\title{
The Bounds for the First General Zagreb Index of a Graph
}

\author{
$\operatorname{Rao} \mathbf{L i}^{1}$ \\ ${ }^{1}$ Department of Mathematical Sciences, University of South Carolina Aiken, Aiken, SC 29801, USA
}

\section{Article Info}

Keywords: The first general Zagreb index, the chromatic number, the clique number, the independent number.

2010 AMS: $05 C 09$

Received: 21 July 2021

Accepted: 22 November 2021

Available online: 30 December 2021

\begin{abstract}
The first general Zagreb index of a graph $G$ is defined as the sum of the $\alpha$ th powers of the vertex degrees of $G$, where $\alpha$ is a real number such that $\alpha \neq 0$ and $\alpha \neq 1$. In this note, for $\alpha>1$, we present upper bounds involving chromatic and clique numbers for the first general Zagreb index of a graph; for an integer $\alpha \geq 2$, we present a lower bound involving the independence number for the first general Zagreb index of a graph.
\end{abstract}

\section{Introduction}

We consider only finite undirected graphs without loops or multiple edges. Notation and terminology not defined here follow those in [2]. Let $G=(V(G), E(G))$ be a graph with $n$ vertices and $e$ edges, where $V=\left\{v_{1}, v_{2}, \ldots, v_{n}\right\}$. We assume that the vertices in $G$ are arranged such that $\Delta(G)=d_{G}\left(v_{1}\right) \geq d_{G}\left(v_{2}\right) \geq \cdots \geq d_{G}\left(v_{n}\right)=\delta(G)$, where $d_{G}\left(v_{i}\right)$, for each $i$ with $1 \leq i \leq n$, is the degree of vertex $v_{i}$ in $G$. The chromatic number, denoted $\chi(G)$, of a graph $G$ is the smallest number of colors which can be assigned to $V(G)$ so that the adjacent vertices in $G$ are colored differently. A clique of a graph $G$ is a complete subgraph of $G$. A clique of largest possible size is called a maximum clique. The clique number, denoted $\omega(G)$, of a graph $G$ is the number of vertices in a maximum clique of $G$. A set of vertices in a graph $G$ is independent if the vertices in the set are pairwise nonadjacent. A maximum independent set in a graph $G$ is an independent set of largest possible size. The independence number, denoted $\beta(G)$, of a graph $G$ is the cardinality of a maximum independent set in $G$. If $H$ is any graph of order $n$ with degree sequence $d_{H}\left(v_{1}\right) \geq d_{H}\left(v_{2}\right) \geq \cdots \geq d_{H}\left(v_{n}\right)$, and if $H^{*}$ is any graph of order $n$ with degree sequence $d_{H}^{*}\left(v_{1}\right) \geq d_{H}^{*}\left(v_{2}\right) \geq \cdots \geq d_{H}^{*}\left(v_{n}\right)$, such that $d_{H}\left(v_{i}\right) \leq d_{H}^{*}\left(v_{i}\right)$ (for each $i$ with $1 \leq i \leq n$ ), then $H^{*}$ is said to dominate $H$. We use $C(n, r)$ to denote the number of $r$-element subsets of a set of size $n$, where $n$ and $r$ are nonnegative integers such that $0 \leq r \leq n$.

The first Zagreb index was introduced by Gutman and Trinajstić in [8]. For a graph $G$, its first Zagreb index is defined as $\sum_{i=1}^{n} d_{G}^{2}\left(v_{i}\right)$. Li and Zheng in [9] further extended the first Zagreb index of a graph and introduced the concept of the first general Zagreb index of a graph. The first general Zagreb index, denoted $M_{\alpha}(G)$, of a graph $G$ is defined as $\sum_{i=1}^{n} d_{G}^{\alpha}\left(v_{i}\right)$, where $\alpha$ is a real number such that $\alpha \neq 0$ and $\alpha \neq 1$.

In this note, we will present upper bounds involving chromatic and cliques numbers for the first general Zagreb index of a graph when $\alpha>1$ and a lower bound involving the independent number for the first general Zagreb index of a graph when $\alpha$ is an integer at least 2. The main results of this note are as follows.

Theorem 1.1. Let $G$ be a graph of order $n$. Assume $\alpha$ is a real number such that $\alpha>1$. Then

(1) $M_{\alpha} \leq n^{2}(n-1)^{\alpha-1}\left(1-\frac{1}{\chi}\right)$.

Equality holds if and only if $G$ is $K_{n}$.

(2) $M_{\alpha} \leq n^{2}(n-1)^{\alpha-1}\left(1-\frac{1}{\omega}\right)$.

Equality holds if and only if $G$ is $K_{n}$. 
Theorem 1.2. Let $G$ be a graph of order $n$. Assume $\alpha$ is an integer which is at least 2. Then

$$
M_{\alpha} \geq \frac{n^{\alpha+1}}{\beta^{\alpha}}+n\left(\Delta^{\alpha}-(1+\Delta)^{\alpha}\right) .
$$

Equality holds if and only if $G$ is a disjoint union of $\beta$ complete graphs of order $\Delta+1$.

\section{Lemmas}

In order to prove Theorem 1 and Theorem 2, we need the following results as our lemmas. The first one is a theorem proved by Erdős in [6]. Its proofs in English can be found in [1].

Lemma 2.1. If $H$ is any graph of order $n$, then there exists a graph $H^{*}$ of order $n$, where $\chi\left(H^{*}\right) \leq \omega(H)$, such that $H^{*}$ dominates $H$.

The second one can be found in [4] and [10].

Lemma 2.2. If $G$ is a graph, then

$$
\beta \geq \sum_{v \in V} \frac{1}{d(v)+1}
$$

Equality holds if and only if each component of $G$ is complete.

\section{Proofs}

Next, we will prove Theorem 1.1. The ideas from the proofs of Theorem 3 on Page 53 in [5] will be used in the proofs of Theorem 1.1 below.

Proof of (1) in Theorem 1.1 Let us partition the vertex set $V$ of $G$ into the pairwise disjoint nonempty subsets of $V_{1}, V_{2}, \ldots, V_{\chi}$ such that $V_{i}$ is independent for each $i$ with $1 \leq i \leq \chi$. Set $\left|V_{i}\right|:=n_{i}$ for each $i$ with $1 \leq i \leq \chi$. Then we have that $n=\sum_{i=1}^{\chi} n_{i}$ and $d(x) \leq n-n_{i}$ for each vertex $x$ in $V_{i}$ and each $i$ with $1 \leq i \leq \chi$. Without loss of generality, we assume that $n_{1} \leq n_{2} \leq \cdots \leq n_{\chi}$. From Cauchy-Schwarz inequality, we have that

$$
\sum_{i=1}^{\chi} n_{i}^{2} \geq \frac{\left(\sum_{i=1}^{\chi} n_{i}\right)^{2}}{\chi}=\frac{n^{2}}{\chi}
$$

Now

$$
\begin{aligned}
M_{\alpha} & =\sum_{v \in V} d^{\alpha}(v), \\
& =\sum_{i=1}^{\chi} \sum_{v \in V_{i}} d^{\alpha}(v) \\
& \leq \sum_{i=1}^{\chi} n_{i}\left(n-n_{i}\right)^{\alpha}, \\
& =\sum_{i=1}^{\chi} n_{i}\left(n-n_{i}\right)\left(n-n_{i}\right)^{\alpha-1} \leq \sum_{i=1}^{\chi} n_{i}\left(n-n_{i}\right)\left(n-n_{1}\right)^{\alpha-1}, \\
& =\left(n-n_{1}\right)^{\alpha-1} \sum_{i=1}^{\chi} n_{i}\left(n-n_{i}\right) \leq(n-1)^{\alpha-1}\left(n^{2}-\sum_{i=1}^{\chi} n_{i}^{2}\right) \leq(n-1)^{\alpha-1}\left(n^{2}-\frac{n^{2}}{\chi}\right)=n^{2}(n-1)^{\alpha-1}\left(1-\frac{1}{\chi}\right) .
\end{aligned}
$$

If

$$
M_{\alpha}=n^{2}(n-1)^{\alpha-1}\left(1-\frac{1}{\chi}\right),
$$

we, from the above proofs, have that $n_{1}=n_{2}=\cdots=n_{\chi}=1$ and $d(v)=n-1$ for each vertex $v$ in $V$. Thus $G$ is $K_{n}$. If $G$ is $K_{n}$, it is easy to verify that

$$
M_{\alpha}=n^{2}(n-1)^{\alpha-1}\left(1-\frac{1}{\chi}\right) .
$$

This completes the proof of (1) in Theorem 1.1.

Proof of (2) in Theorem 1.1 From Lemma 2.1, we can find a graph $G^{*}$ dominating $G$ and $\chi\left(G^{*}\right) \leq \omega(G)$. From (1) of this theorem, we have that

$$
M_{\alpha}(G) \leq M_{\alpha}\left(G^{*}\right) \leq n^{2}(n-1)^{\alpha-1}\left(1-\frac{1}{\chi\left(G^{*}\right)}\right) \leq n^{2}(n-1)^{\alpha-1}\left(1-\frac{1}{\omega(G)}\right) .
$$

If

$$
M_{\alpha}(G)=n^{2}(n-1)^{\alpha-1}\left(1-\frac{1}{\omega}\right)
$$


then

$$
M_{\alpha}\left(G^{*}\right)=n^{2}(n-1)^{\alpha-1}\left(1-\frac{1}{\chi\left(G^{*}\right)}\right)
$$

From (1) of this theorem, we have that $G^{*}$ is $K_{n}$ and $\chi\left(G^{*}\right)=n$. Thus $\omega(G) \geq \chi\left(G^{*}\right)=n$. Hence $G$ is $K_{n}$. If $G$ is $K_{n}$, it is again easy to verify that

$$
M_{\alpha}(G)=n^{2}(n-1)^{\alpha-1}\left(1-\frac{1}{\omega}\right) .
$$

This completes the proof of (2) in Theorem 1.1.

Next, we will prove Theorem 1.2 which is motivated by Theorem 3.1 on Page 309 in [7].

Proof of Theorem 1.2 From Lemma 2.2 and the inequalities on the power means, arithmetic means, and harmonic means of $n$ positive integers, we have that

$$
\left(\frac{\left(1+d_{1}\right)^{\alpha}+\left(1+d_{2}\right)^{\alpha}+\cdots+\left(1+d_{n}\right)^{\alpha}}{n}\right)^{\frac{1}{\alpha}} \geq \frac{\left(1+d_{1}\right)+\left(1+d_{2}\right)+\cdots+\left(1+d_{n}\right)}{n} \geq \frac{n}{\frac{1}{1+d_{1}}+\frac{1}{1+d_{2}}+\cdots+\frac{1}{1+d_{n}}} \geq \frac{n}{\beta} .
$$

Then

$$
\left(1+d_{1}\right)^{\alpha}+\left(1+d_{2}\right)^{\alpha}+\cdots+\left(1+d_{n}\right)^{\alpha} \geq \frac{n^{\alpha+1}}{\beta^{\alpha}} .
$$

It is easy to check that for each $i$ with $1 \leq i \leq n$ we have

$$
\left(1+d_{i}\right)^{\alpha}=\sum_{k=0}^{\alpha} C(\alpha, k) d_{i}^{k} \leq \sum_{k=0}^{\alpha} C(\alpha, k) \Delta^{k}-\Delta^{\alpha}+d_{i}^{\alpha}=(1+\Delta)^{\alpha}-\Delta^{\alpha}+d_{i}^{\alpha} .
$$

Equality holds if and only if $d_{i}=\Delta$. Thus

$$
(1+\Delta)^{\alpha}-\Delta^{\alpha}+d_{1}^{\alpha}+(1+\Delta)^{\alpha}-\Delta^{\alpha}+d_{2}^{\alpha}+\cdots+(1+\Delta)^{\alpha}-\Delta^{\alpha}+d_{n}^{\alpha} \geq\left(1+d_{1}\right)^{\alpha}+\left(1+d_{2}\right)^{\alpha}+\cdots+\left(1+d_{n}\right)^{\alpha} \geq \frac{n^{\alpha+1}}{\beta^{\alpha}} .
$$

Therefore

$$
M_{\alpha} \geq \frac{n^{\alpha+1}}{\beta^{\alpha}}+n\left(\Delta^{\alpha}-(1+\Delta)^{\alpha}\right) .
$$

If

$$
M_{\alpha}=\frac{n^{\alpha+1}}{\beta^{\alpha}}+n\left(\Delta^{\alpha}-(1+\Delta)^{\alpha}\right),
$$

then $d_{1}=d_{2}=\cdots=d_{n}=\Delta$. From Lemma 2, we have that $G$ is a union of $\beta$ complete graphs of order $\Delta+1$. If $G$ is a union of $\beta$ complete graphs of order $\Delta+1$, then $(\Delta+1) \beta=n$. It is easy to verify that

$$
M_{\alpha}=\frac{n^{\alpha+1}}{\beta^{\alpha}}+n\left(\Delta^{\alpha}-(1+\Delta)^{\alpha}\right) .
$$

This completes the proof of Theorem 1.2.

Remark 3.1. Let $G$ be a graph with $n$ vertices and e edges. Notice that

$$
n+4 e+M_{2}=\sum_{i=1}^{n}\left(1+d_{i}\right)^{2} \geq \frac{n^{3}}{\beta^{2}} .
$$

We have that

$$
M_{2} \geq \frac{n^{3}}{\beta^{2}}-n-4 e
$$

It can be verified that $M_{2}=\frac{n^{3}}{\beta^{2}}-n-4 e$ if and only if $G$ is a disjoint union of $\beta$ complete graphs of order $\Delta+1$.

Remark 3.2. Let $G$ be a graph with $n$ vertices and e edges. Notice that

$$
n+6 e+3 M_{2}+M_{3}=\sum_{i=1}^{n}\left(1+d_{i}\right)^{3} \geq \frac{n^{4}}{\beta^{3}} .
$$

We have that

$$
M_{3} \geq \frac{n^{4}}{\beta^{3}}-n-6 e-3 U
$$

where $U$ is an upper bound for $M_{2}$. A variety of concrete expressions for $U$ can be found in [3]. 


\section{Acknowledgements}

The author would like to thank the referees for their suggestions and comments which improve the original version of the paper.

\section{Funding}

There is no funding for this work.

\section{Availability of data and materials}

Not applicable.

\section{Competing interests}

The authors declare that they have no competing interests.

\section{Author's contributions}

All authors contributed equally to the writing of this paper. All authors read and approved the final manuscript.

\section{References}

[1] B. Bollobás, Extremal Graph Theory, Academic Press, London (1978), Pages 295 and 296.

[2] J.A. Bondy, U.S.R. Murty, Graph Theory with Applications, Macmillan, London and Elsevier, New York (1976).

[3] B. Borovićanin, K. Das, B. Furtula, I. Gutman, Bounds for Zagreb indices, MATCH Commun. Math. Comput. Chem. 78 (2017), 17-100.

[4] Y. Caro, New results on the independence number, Technical Report, Tel-Aviv University, 1979.

[5] C. S. Edwards and C. H. Elphick, Lower bounds for the clique and the chromatic numbers of a graph, Discrete Appl. Math., 5 (1983,) 51-64.

[6] P. Erdős, On the graph theorem of Turán (in Hungarian), Mat. Lapok, 21 (1970), 249-251.

[7] S. Filipovski, New bounds for the first Zagreb index, MATCH Commun Math Comput Chem., 54 (2005), 195-208

[8] I. Gutman and N. Trinajstić, Graph theory and molecular orbitals, total $\pi$-electron energy of alternant hydroncarbons, Chem. Phys. Lett., 17 (1972), $535-538$

[9] X. Li and Z. Zheng, A unified approach to the extremal trees for different indices, MATCH Commun. Math. Comput. Chem,. 85(2021), 303-312.

[10] V. K. Wei, A lower bound on the stability number of a simple graph, Bell Laboratories Technical Memorandum, No. 81-11217-9, 1981. 